\title{
Meconium ileus equivalent in adults with cystic fibrosis of pancreas: a report of six cases
}

\author{
MARGARET E HODSON， MARGARET B MEARNS， J C BATTEN
}

British Medical fournal, 1976, 2, 790-791

\section{Summary}

Eleven episodes of "meconium ileus equivalent" have been seen in six adults with cystic fibrosis of the pancreas. Three patients were initially treated surgically; one died and the other two developed serious postoperative chest infections. Six episodes were successfully treated medically with acetylcysteine orally and by enema, nasogastric suction, and intravenous fluids. Op ation should be avoided if possible, and maintenance treatment with acetylcysteine may be necessary to prevent relapse.

\section{Introduction}

"Meconium ileus equivalent" is the term used to describe intestinal obstruction in patients with cystic fibrosis when the obstruction occurs outside the neonatal period. Early and late intestinal complications of cystic fibrosis are due to solid or semi-solid contents being present in the terminal ileum, where the faecal stream is normally liquid. In older patients faecal masses may be discovered on routine examination; they are usually mobile and non-tender, and are found in the right iliac fossa. In more severe cases intestinal obstruction develops, with colicky abdominal pain, vomiting, and constipation. An abdominal film shows small bowel obstruction. This condition is meconium ileus equivalent.

We report here 11 episodes of meconium ileus equivalent which presented as intestinal obstruction in six adults with cystic fibrosis of the pancreas.

\section{Patients and methods}

Eighty-five patients with cystic fibrosis, all over 15 years of age, were attending the outpatient department at Brompton Hospital between 1971 and 1974. Six developed intestinal obstruction. All had been diagnosed as having cystic fibrosis in early childhood, when they had developed recurrent chest infections and malabsorption. They were subsequently found to have sweat sodium concentrations above $70 \mathrm{mmol}(\mathrm{mEq}) / \mathrm{l}$. All were on regular treatment with pancreatic enzymes, physiotherapy, and antibiotics as indicated.

Medical treatment for obstruction consisted of intravenous fluids and intermittent nasogastric aspiration together with $30 \mathrm{ml}$ of a $20^{\circ}$ solution of acetylcysteine three times a day via the nasogastric tube and $30 \mathrm{ml}$ acetylcysteine in normal saline as an enema three times a day. As the obstruction resolved the frequency of the enemas was reduced and patients were kept on a dose of $10-20 \mathrm{ml}$ acetylcysteine orally three times a day, the taste being partially disguised with concentrated orange or blackcurrant juice.

\section{Case reports}

Case 1-A 23-year-old man developed subacute intestinal obstruction with faecal impaction that was treated conservatively by enema in 1967.

\section{Brompton Hospital, London SW3 6HP}

MARGARET E HODSON, MB, MRCP, senior medical registrar

MARGARET B MEARNS, DCH, MRCP, consultant paediatrician

J C BATTEN, MD, FRCP, consultant physician
Four years later he was operated on at another hospital for intestinal obstruction. The last 18 inches $(45 \mathrm{~cm})$ of small bowel were found to contain hard impacted faeces which were removed through an enterostomy. After operation he developed respiratory failure and died. At the age of 22 he had developed diabetes, which was controlled by lente insulin.

Case 2-A 26-year-old man was admitted to another hospital in 1971 with 48 hours' colicky abdominal pain and constipation. Abdominal radiographs showed fluid levels in the small bowel, and intestinal obstruction was diagnosed. At operation there was free fluid in the peritoneal cavity and distension of the proximal small bowel. The terminal $90-\mathrm{cm}$ of ileum was grossly hypertrophied and contained much sticky, yellow, inspissated material. The bowel was opened, irrigated, and an ileotransverse colostomy performed. After operation he developed sputum retention and was transferred to Brompton Hospital for intensive chest treatment. He eventually recovered and was maintained on oral acetylcysteine with no further bowel trouble, but he died of respiratory failure in March 1974. He had been a diabetic requiring insulin since the age of 14 .

Case 3-A 23-year-old man developed colicky abdominal pain and constipation in February 1973. His abdomen was distended, there was a mass in the right iliac fossa and the rectum was empty. (The appendix had been removed previously.) An abdominal film showed dilated loops of small bowel with fluid levels and considerable faecal matter in the colon. He was treated with acetylcysteine by enema and nasogastric tube, together with intravenous fluids. The obstruction resolved and he was discharged taking oral acetylcysteine three times a day. He disliked the taste and stopped treatment. In June 1973 symptoms of obstruction recurred and resolved once more with acetylcysteine. In September 1973 he developed diabetes mellitus and a month later after stopping acetylcysteine again he developed intestinal obstruction. This resolved on treatment, and after this third episode he took acetylcysteine regularly and remained free of abdominal symptoms until his death from respiratory failure in February 1974.

Case 4-A 21-year-old man was admitted elsewhere in 1968 with abdominal pain, vomiting, and constipation of three days' duration. An abdominal radiograph showed fluid levels in the small bowel. At laparotomy free fluid was found in the peritoneum and the surgeon recorded that "the ileum was filled with inspissated, putty-like material. The bowel was distended proximally and collapsed distally; the transverse and sigmoid colon were normal." Some of the inspissated material was removed and he was subsequently treated with enemas and intravenous fluids. He developed a postoperative chest infection but recovered. In July 1973 a further attack of intestinal obstruction necessitated admission to Brompton Hospital. The abdominal radiographs are shown in the figure. He was treated with intravenous fluids and acetylcysteine by mouth and enema, and the obstruction resolved. Oral acetylcysteine was continued and he had no further bouts of abdominal pain. In October 1974, however, after stopping treatment, he again had an attack of intestinal obstruction which responded to acetylcysteine. He has continued on treatment since and there have been no abdominal symptoms. He had been an insulin-dependent diabetic since the age of 18 .

Case 5-A 25-year-old man had a five-day history of nausea, vomiting, colicky abdominal pain, and constipation when seen in February 1974. An abdominal radiograph showed small bowel obstruction with fluid levels. He was treated with intravenous fluids and acetylcysteine by nasogastric tube and enema. Within three days the obstruction had resolved and the enemas were stopped. He has been maintained on oral acetylcysteine and has had no further abdominal pain; no masses have been palpable in the abdomen.

Case 6-A 21-year-old woman had an episode of intestinal obstruction treated surgically at the age of 4 but no details were available. She had minimal respiratory symptoms during her teens and required only intermittent antibiotics; lung function was normal apart from some hyperinflation in February 1974. A month later she complained of bouts of severe intermittent abdominal pain for five days, accompanied by constipation and vomiting. The abdomen was distended and there was a palpable mass related to the surgical scar. An abdominal radiograph showed fluid levels in the small bowel. She was treated with intravenous fluids and acetylcysteine orally and by enema and her acute symptoms subsided. Over the next few weeks she continued to complain of colicky abdominal pain and a mass again became palpable. Because of the possibility of adhesions a laparotomy was performed and small bowel obstruction due to thickening and inflammation of the terminal ileum was found. A right hemicolectomy was performed, the tissue being extremely friable. Bleeding was difficult to control and she died after operation.

\section{Discussion}

Ten to twenty per cent of patients with cystic fibrosis develop intestinal obstruction (meconium ileus) in the neonatal period 

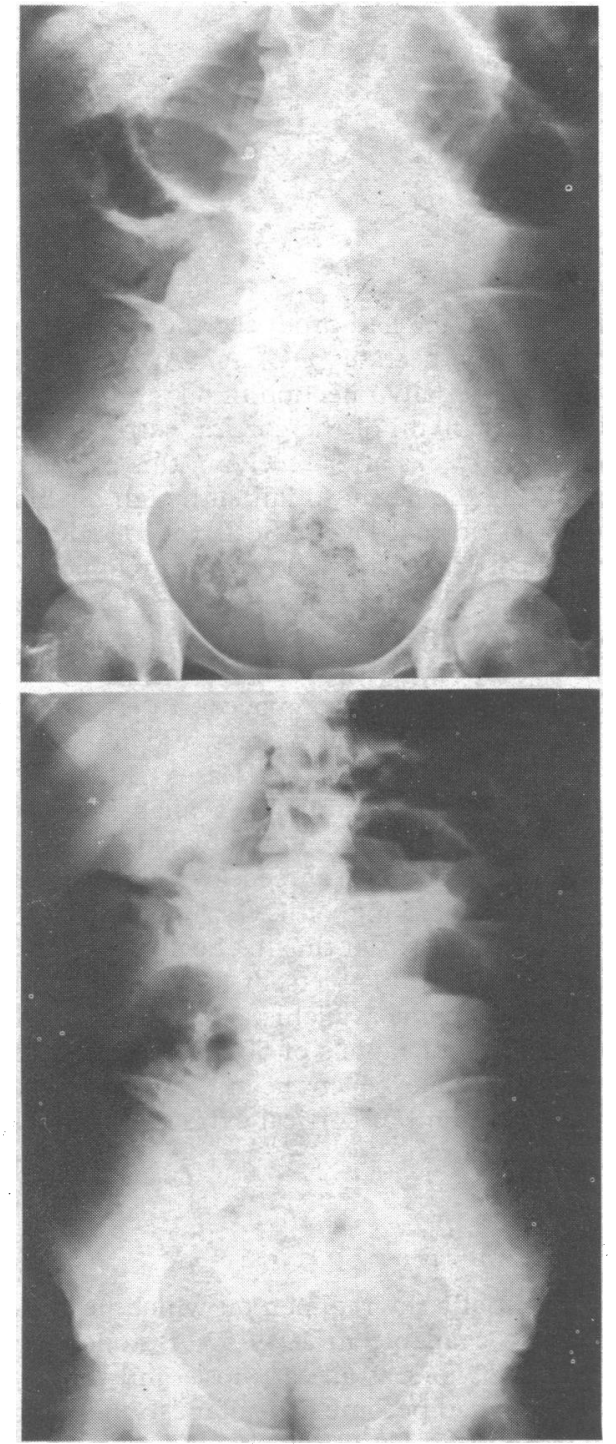

Both supine (top) and erect (bottom) abdominal radiographs show distended bowel with air fluid levels. There is much faecal material in colon. Appearances are those of small bowel obstruction.

and require surgical treatment. ${ }^{1}$ The incidence of obstruction in older patients is unknown. Levy ${ }^{2}$ reported a boy aged 7 months and Fisher ${ }^{3}$ a boy aged 13 months who developed intestinal obstruction. Faconi ${ }^{4}$ reported three cases of obstruction at the age of $2 \frac{1}{2}$ years, 2 years 10 months, and 20 years among a clinic population of 158 children. Twenty-six cases of intestinal obstruction occurring outside the neonatal period were reviewed ${ }^{5}$ in a report describing a 20-year-old man who developed meconium ileus equivalent and died after surgery and attempts at relieving the obstruction with nasogastric suction and pancreatic enzymes.

The cause of intestinal obstruction is uncertain. About $80 \%$ of patients with cystic fibrosis have complete loss of pancreatic enzyme activity, causing malabsorption, ${ }^{6}$ and the intestinal content will be altered as a result. Abnormal intestinal mucoproteins $^{7}$ and an excess of tenacious intestinal mucus ${ }^{8}$ have been found, but infants with muconeum ileus had no more severe disease than those without. ${ }^{9}$ The intestinal glands of the small bowel, however, were more hypertrophied and contained considerable amounts of secretions. Hallberg ${ }^{10}$ described a 15 year old with obstruction, whose intestinal mucosa was coated with 3-4 mm of thick mucilaginous membrane, and attempts to remove this gave rise to numerous punctate haemorrhages (see case 6). In three other older patients ${ }^{11}$ who required surgery for faecal retention the outstanding feature was a heavy layer of adherent mucus overlying a flattened mucosa.

Various conservative measures such as nasogastric lavage and treatment with pancreatic enzymes, and enemas have been tried, but operation has often been necessary. ${ }^{51213}$ Acetylcysteine enemas were suggested by Snyder et al, ${ }^{11}$ and a successful outcome after oral acetylcysteine has been reported in six patients with recurrent abdominal pain and palpable adbominal masses. Their intestines were not, however, completely obstructed. ${ }^{14} 15$ Six of the seven episodes of obstruction treated with acetylcysteine among the present group of patients subsided, and in two cases relapse occurred after treatment was stopped. The importance of continuing treatment after one bout of meconium ileus equivalent was not recognised, and the patients had to be persuaded to continue with the medicine, which is rather unpleasant to take. When obstruction is unrelieved by medical measures, resort to surgery is, of course, necessary; but the dangers of early operation are apparent. One patient died and the other two developed severe postoperative chest infections. The last patient (case 6) was operated on after failing to respond to acetylcysteine and died of blood loss, infection, and failure of wound healing.

Four of the six patients had diabetes, a well-recognised complication of cystic fibrosis in older patients. ${ }^{18}$ The incidence of diabetes appears to increase with age, and whether there is any relation between it and meconium ileus equivalent remains to be seen.

We should like to thank $\operatorname{Dr} G$ Simon for reporting the radiographs and Miss L Topping and Miss G Smith for secretarial help.

ADDENDUM-Since this paper was prepared a further case of intestinal obstruction has been encountered in an 18-year-old with cystic fibrosis. Bleeding was uncontrollable during surgical attempts to relieve the obstruction and replacement treatment with a wide variety of clotting factors did not prevent her dying soon after operation.

\section{References}

i Donnison, A B, Schwachnan, H, and Gross, R E, Pediatrics, 1966, 37, 833.

2 Levy, E, Archives of Diseases of Childhood, 1951, 26, 335.

3 Fisher, O D, Archives of Diseases of Childhood, 1954, 29, 262.

4 Fanconi, A, Helvetica Paediatrica Acta, 1960, 15, 566.

5 Hunton, D B, Long, W K, and Tsumagori, H Y, Gastroenterology, 1966, $50,99$.

6 Kopel, F B, Current Clinical Concepts, 1972, 62, 483.

7 di Sant' Agnese, P A, Dische, Z, and Danilczenko, A, Pediatrics, 1957, $19,252$.

8 Johnson, P G, Biochemistry fournal, 1963, 87, 63.

9 Thomaidis, T S, and Arey, J B, Fournal of Pediatrics, 1963, 63, 444.

10 Hallberg, D, Acta Chirurgica Scandinavica, 1964, 128, 201.

11 Snyder, W H, et al, Pediatrics, 1964, 34, 72.

12 Garnham, J R, and Carter, T R G, Gut, 1964, 5, 256.

13 Mullins, F, Talamo, R, and di Sant' Agnese, P A, fournal of the American Medical Association, 1965, 192, 741.

14 Lillibridge, C B, Docter, J M, and Eidelman, S, fournal of Pediatrics, $1967,71,887$.

15 Gracey, M, Burke, U, and Anderson, C M, Archives of Diseases of Childhood, 1969, 44, 404 .

16 Hardwerger, S, et al, New England fournal of Medicine, 1969, 281, 451. 